\title{
Corela
}

Cognition, représentation, langage

HS-5 | 2006

Organisation des textes et cohérence des discours

\section{Anaphores prédicatives démonstratives : de la cohésion syntagmatique à la cohérence textuelle}

Catherine Schnedecker

\section{OpenEdition}

\section{Journals}

Édition électronique

URL : http://journals.openedition.org/corela/1437

DOI : $10.4000 /$ corela. 1437

ISSN : $1638-573 \mathrm{X}$

Éditeur

Cercle linguistique du Centre et de I'Ouest - CerLICO

Référence électronique

Catherine Schnedecker, «Anaphores prédicatives démonstratives : de la cohésion syntagmatique à la cohérence textuelle », Corela [En ligne], HS-5 | 2006, mis en ligne le 27 octobre 2006, consulté le 02 mai 2019. URL : http://journals.openedition.org/corela/1437 ; DOI : 10.4000/corela.1437

Ce document a été généré automatiquement le 2 mai 2019.

\section{(c) (1) (3) (-)}

Corela - cognition, représentation, langage est mis à disposition selon les termes de la licence Creative Commons Attribution - Pas d'Utilisation Commerciale - Partage dans les Mêmes Conditions 4.0 International. 


\title{
Anaphores prédicatives démonstratives : de la cohésion syntagmatique à la cohérence textuelle
}

\author{
Catherine Schnedecker
}

\section{NOTE DE L'AUTEUR}

Nous remercions chaleureusement G. Kleiber pour ses remarques constructives à la lecture d'une première version de cet article ainsi que D. Legallois et M. Salles pour leur relecture attentive de cette version et leurs suggestions tout à fait opportunes. Les erreurs qui subsistent sont bien évidemment de notre fait.

\section{Introduction}

1 Notre étude portera sur des séquences anaphoriques du type de (1) à (3), bien repérées dans la littérature sur l'anaphore du fait de trois caractéristiques :

1) Un chasseur est arrivé hier ; cet Allemand a manqué tous ses tirs (Milner ${ }^{1}, 1982$, 24, ex. 7a)

2) Il y avait sur la table un livre. Ce torchon... (Corblin, 1987, 210)

3) Mais voilà qu'un beau matin, une vendeuse les sortit de leur boîte pour les essayer à une dame. La dame/l'aimable cliente/cette dame/cette bourgeoise mal attifée/elle les mit, fit quelques pas avec ... (Reichler-Béguelin, 1995, 54, d'après Gripari)

2 1) l'anaphorique s'y exprime très fréquemment - quoique non exclusivement ${ }^{2}-$ par un SN démonstratif, qui peut se composer d'un $\mathrm{N}$ « nu » (1) et (2) ou expansé, dont la tête lexicale est éminemment variable (cf. 4 et 5), ou d'une relative en celui/celle qui (6) : 
4) Il (= J. Villeret) y incarnait François Pignon, l'humble fonctionnaire humilié puis triomphant, un rôle qui lui valut le César du meilleur acteur en 1999. Avec plus de neuf millions d'entrées, le film reste l'un des plus grands succès de l'histoire du cinéma français et a fixé durablement l'image de cet homme un peu trop gros (quoique sa silhouette ait souvent varié au fil des ans), aux grands yeux naïfs, si gentil qu'il parvient à surmonter la méchanceté du reste de l'humanité. Mais ce n'est pas rendre justice à cet acteur étonnant que de se limiter à cette version-là de l'acteur. (Le Monde, 02/02/05)

5) Pour supporter, pour respirer, Sophie Talneau a tenu le journal de sa mise à l'index. C'est drôle et piquant, quotidien et naïf, pertinent et vachard. Au ramdam idéologique, cette Bridget Jones d'ANPE préfère les notations incisives et cruelles. (Libération)

6) Ce dernier (= Yo-Yo Ma) lui (= Erwan Fauré) fit même, un jour de juin 1991, la surprise de venir de Boston jouer pour lui, à Pleyel, le Concerto de Schumann. Le virtuose tenait à saluer celui qui, atteint par la limite d'âge, devait d'un coup quitter le Conservatoire national supérieur de musique de Paris et sa place de violoncelle solo à l'Orchestre national d'Ile-de-France, qu'il avait contribué à créer, en 1974. (Le Monde, 26/06/03)

3 2) ces SN démonstratifs délivrent sur le référent des informations totalement inédites, qu'on ne peut reconstruire ni à partir du segment-source, ni, le cas échéant (cf. 1), du cotexte inséré entre les deux expressions référentielles ;

4 3) ce genre d'expressions, démonstratives ou définies comme en (7), sont de ce fait assimilées à une prédication : témoin le commentaire de Sériot ci-dessous à propos de (7) :

7) Gustave Flaubert naquit à Rouen en 1821. Le père du naturalisme... (Sériot, 1988 :

150)

[...] sous l'apparence d'une simple reprise, d'une simple substitution, on voit que la deuxième mention est ici une prédication. Mais une prédication implicite, en ce que la répétition, la rénomination, qui est en principe un facteur de cohérence textuelle (la « trame textuelle »), masque en fait une adjonction d'information, qui permet de faire avancer le texte dans une nouvelle direction. (P. Sériot, art. cit. : 151 ; les gras sont de nous)

D'où l'étiquette de SN démonstratifs prédicatifs (SNDP) que nous utiliserons désormais pour les qualifier.

Les séquences de ce type posent bon nombre de problèmes ${ }^{3}$ dont certains impliquent les notions de cohésion/cohérence. Le premier tient à leur recevabilité. Parfaitement admises par leurs auteurs, ainsi que le suggère le propos ci-dessous, elles sont mises à l'index par d'autres (p.e. Kleiber, 1998 : 58 et 65 et nos propres informateurs):

[...] nous tenons en tout cas à souligner que la prédication assurée par le biais du SN démonstratif n'est pas, ou du moins n'est pas forcément prédéterminée codiquement. Si tel était le cas, on serait conduit à considérer comme quasi délictueuses les recatégorisations innovantes ou paradoxales et les métaphores d'invention supportées par le SN démonstratif, alors qu'elles font partie du fonctionnement le plus normal de cet outil linguistique (Reichler-Béguelin, 1995, $57)^{4}$

7 De fait, leur occurrence attente à la cohésion référentielle d'abord, dans la mesure où les ruptures ou changements discursifs opérés par les SN démonstratifs en général ont été mis en évidence par Kleiber (1984, 1986, 1994, entre autres) et De Mulder (1997, entres autres). Ensuite, leur «nouveauté informative » les rend difficilement prédictibles dans les modèles de la cohésion lexicale défendus par Halliday \& Hasan et Hoey, présentés par Legallois (2004), fondés sur le phénomène de la répétition informationnelle, littérale ou non. Enfin, certains SNDP semblent sérieusement mettre en question la cohérence 
textuelle. C'est ainsi que les SN ce virtuose de l'accordéon de (8) et ce Normand... de (9) sont, à nos yeux du moins, problématiques étant donné qu'on voit mal quel rapport établir, d'une part, entre le fait d'être un virtuose de l'accordéon et celui de déménager tous les deux ans, et, d'autre part, celui de savoir précocément ce que l'on veut faire de sa vie et de descendre d'une famille de commerçants normands :

8) Daniel Libeskind

Bâtisseur d'espoir

Fils d'immigrés juifs polonais, cet architecte a remporté le concours pour la reconstruction du World Trade Center.

Né en Pologne après la Seconde Guerre Mondiale, Daniel Libeskind y a vécu l'antisémitisme du régime communiste après que ses parents avaient subi celui des nazis. Sa famille a rapidement émigré en Israël, où elle s'est vite lassée de la vie dans les kibboutzim avant de partir pour les États Unis. De quoi donner le goût du nomadisme - avec quatorze déménagements en trente-cinq ans! - à ce virtuose de l'accordéon! (TV Hebdo, 2005)

9) Il (= Dominique Besnehard) est ce personnage incontournable du cinéma français qui monte les marches de Cannes au bras des stars dont il est l'agent [...]. La consécration d'un rêve de môme.

À 5 ans, ce Normand issu d'une famille de commerçants d'Houlgate sait déjà ce qu'il fera dans la vie [...]. (Marie-Claire, mai 2005)

De là, la double attitude face aux SNDP : l'une très accueillante et permissive qui pose que les usages du démonstratif sont entièrement libres, et qu'illustrent les citations cidessous :

[...] une opération interprétative régulièrement associée à l'usage du démonstratif, et théoriquement «libre»; une succession quelconque un $\mathrm{N}_{\mathrm{i}} \ldots$ ce $\mathrm{N}_{\mathrm{j}}$ est régulièrement interprétable comme désignation de $\mathrm{N}_{\mathrm{i}}$ reclassifié comme $\mathrm{N}_{\mathrm{j}}$, et il paraît très difficile de circonscrire sur des bases strictement linguistiques, un ensemble de reclassification qui seraient prohibées. Les reclassifications peuvent seulement être évaluées, linguistiquement comme mise en rapport de référence virtuelle : 1) ce $\mathrm{N}_{j}$ désigne l'objet $\mathrm{x}$ précédemment construit comme $\mathrm{N}_{\mathrm{i}}$;2) il opère la reclassification d'un $\mathrm{N}_{\mathrm{i}}$ comme $\mathrm{N}_{\mathrm{j}}$ (Corblin, 1987, 212 ; nous soulignons)

[...] dans bien des cas, l'énonciateur se trouve devant une série d'alternatives, et, dans la mesure où l'identification de l'objet-de-discours visé n'est pas compromise, il est libre de sélectionner et de formater à sa guise l'expression référentielle. » (Reichler-Béguelin 1995, 54)

[...] L'exemple < cf. (5) dans notre numérotation >, montre bien qu'il n'existe pas de contrainte de cooccurrence stricte entre un "antécédent » donné et une certaine forme de rappel [...] (Reichler-Béguelin art. cit., 58)

Une autre, partant des difficultés, sinon les réactions, que suscitent les énoncés (2) et (8-9) tendrait à penser que les SNDP ne s'emploient pas aussi librement qu'il est dit mais qu'ils sont au contraire assujettis à des contraintes que nous nous attacherons, dans la suite de notre propos, à rappeler, discuter ou dégager. C'est ainsi que nous en passerons deux en revue : les unes internes à la prédication liée aux SNDP, les autres qui dépendent de leur environnement discursif, les unes impliquant autant que les autres des phénomènes de cohésion et/ou de cohérence textuelle. Notre propos s'appuiera sur un corpus ouvert et réalisé de manière tout à fait artisanale de " portraits » journalistiques tirés des quotidiens Libération et Le Monde. 


\section{Contraintes « internes » aux SN démonstratifs prédicatifs}

10 Les conceptions relativement permissives évoquées dans notre introduction ont été abondamment commentées, discutées et modulées notamment par Kleiber (1984, 1988, entre autres) et Marandin (1986) dont les travaux font ressortir une contrainte primordiale que nous résumons très succinctement.

\subsection{UNE RELATION DE TYPE ÊTRE ENTRE LES N DES SNDP ET DE LEUR SOURCE (MARANDIN, 1986 ; KLEIBER, 1988)}

11 La première fait intervenir la nécessité d'une relation attributive entre le SND et son antécédent. Celle-ci s'exprime diversement selon les auteurs. Elle est très stricte et exigeante sur le rapport entre la source et le $\mathrm{N}$ du SND chez Marandin (1986) et rigoureusement conditionnée par le sémantisme du $\mathrm{N}^{5}$ :

L'interprétation anaphorique d'un SN démonstratif avec pour nom recteur certains substantifs, requiert l'occurrence effective dans le contexte gauche d'un terme avec lequel ils peuvent être mis en relation par une relation « être-X » (Marandin, 1986, 77) 6

Pour Kleiber $(1984,1988)$, elle provient du sens même du démonstratif :

Une description démonstrative ce $\mathrm{N}$, anaphorique ou non, équivaut à une structure attributive classificatoire présupposée du type Ce $\mathrm{N}$ est un/du N (ou N) (Kleiber, $1984 ; 1988,62)$

13 Avec comme conséquence que :

Le rôle crucial du nom recteur est donc de limiter le domaine des référents possibles à ceux qui admettent d'être nommés ou classés comme des N. (Kleiber 1988, 62)

Elle est en outre, de l'aveu même de l'auteur et, par contraste avec celle de Marandin (1986) :

[...] beaucoup moins stricte en ce qu'elle n'exige pas une paraphrase correspondant à une phrase copulative mettant en relation le terme anaphorique et un élément du cotexte. Le rapport attributif postulé ne s'établit pas entre un élément du contexte (ou le contexte dans sa globalité) et la description anaphorique mais entre le référent conçu comme non classifié ou non nommé et le substantif recteur (Kleiber, $1988: 63)$

15 Cette première contrainte présente un double avantage. D'abord, elle fait le départ entre les SNDP liés sémantiquement à leur source, comme dans (10) et (11), et ceux qui ne le sont pas :

10) Il y avait sur la table un roman. Ce livre... (Kleiber, 1988, 65)

10a) Le $x$ identifié comme un roman est un livre

11) (?) Il y avait sur la table un livre. Ce roman... (Corblin, 1987, 210, l'indexation est

de G. Kleiber, 1988, 62)

11a) Le $x$ identifié comme un livre est un roman

D'autre part, elle rend compte des différences entre (10) et (11) et notamment du rôle de la hiérarchie-être qui s'applique dans (10) et mais pas dans (11) à propos duquel G. Kleiber explique que : 
Les traits analytiques et stéréotypiques de livre ne comportent pas le trait être un roman qui serait nécessaire pour opérer une saisie référentielle anaphorique : un livre peut être un roman et c'est tout [...]. (Kleiber, 1988, 64) telle celle qu'établit ce torchon dans (4) où l'anomalie provient, comme l'explique Kleiber $(1988,65)$, de ce que manque l'assertion métaphorique intermédiaire comme quoi (Les livres sont des torchons) :

4) Il y avait sur la table un livre. Ce torchon... (F. Corblin, 1987, 210)

4a) ? Le x identifié comme un livre est un torchon

On comprend dès lors pourquoi (1) pose problème. D'une part, il ne préexiste pas de relation sémantique entre chasseur et allemand, qui n'est en rien un trait prototypique du $\mathrm{N}$ de chasseur. D'autre part, rien dans l'environnement de la $\mathrm{p}_{1}$ ne fournit pas plus de prédication visant à reclassifier le référent qu'il n'apporte de solution à ce problème classificatoire :

? Le x identifié comme un chasseur est (un) Allemand

\subsection{Le type de copulative sous-jacente (Van Peteghem, 1998)}

La relation être jouerait donc ainsi un rôle primordial sur la teneur lexicale de l'anaphore démonstrative. Tout dépend alors, selon Van Peteghem (1998), du type de relation copulative qu'il est possible de reconstruire pour unir le SN-source et le SN2 coréférant, et qui serait étroitement corrélé à la nature définie vs démonstrative du SN2 (cf. schéma i) :

i) Type de copule $<\mathrm{SN} 1$ être SN2 $>\rightarrow \mathrm{SN} 1 \ldots$ (ce vs le) N2

\subsubsection{Des copules identificationnelles ou prédicationnelles...}

21 C'est ainsi que la reclassification reposant sur une phrase d'identité ${ }^{7}$ et qui constituerait, pour cette raison, une description autonome, n'aboutirait pas facilement à un SN démonstratif mais plutôt à un SN défini, comme le suggère l'exemple (12) emprunté à l'auteure :

12) Jacques Chirac est arrivé à Bruxelles. (\#Ce+le) président de la République française rencontrera ce matin le premier ministre belge (Van Peteghem, 1998, son ex. 11)

- Jacques Chirac est le président de la République française : copule d'identité Les prédications sous-jacentes, susceptibles de donner lieu à un SN démonstratif seraient, selon l'auteur, les copules dites : «identificationnelles » «où l'attribut identifie le référent du sujet, sans toutefois être pleinement référentiel » (art.cit., 572) ${ }^{8}$, comme dans (13) que l'auteur emprunte à Kleiber (1988:66) :

13) À 60 ans, Paul Dubois se retira dans son village. Cet ancien coureur cycliste ...

(Kleiber, 1988 : 66, cité par Van Peteghem, 1998 : 573, son ex. 13) 
- Paul Dubois est un ancien coureur cycliste: relation copulative identificationnelle ${ }^{9}$ .) et qui valent pour les SN démonstratifs ainsi que «dans certains cas pour le défini ». C'est ce qu'illustrerait (14) :

14) Jean est arrivé en retard. $<L^{\prime}+C e t>$ imbécile avait raté une fois de plus son train de 8 heures. (Ex. 14, de M. Van Peteghem, ibid., nous ajoutons le démonstratif)

- Jean est un imbécile : relation copulative prédicationnelle

\subsubsection{DONT LE SN ATTRIBUTIF SERAIT DE FORME UN $N$ +MODIfIEUR}

Partant du propos de M. Van Peteghem, on peut se demander quelle est la forme du SN « attribut » qui donne préférentiellement lieu aux SNDP.

L'observation de notre corpus révèle que l'écrasante majorité des SNDP se constitue de SN modifiés, selon des procédés divers. En tout cas, les SNDP «nus » sont très rares ${ }^{10}$. Ils se limitent pour l'essentiel aux N de nationalité/d'appartenance ethnique comme dans (14) et (15) :

14) Hubert Sauper, un œil sur ce bas monde

Dans "Le Cauchemar de Darwin", le réalisateur autrichien utilise l'image comme argument pour dénoncer la mondialisation. Il maîtrise l'art de frapper en finesse, sans commentaire ni jugement.

Y a-t-il un fond au puits de l'abjection? A la question, Hubert Sauper répond caméra au poing. Ou plutôt avec une caméra en guise de poing. Pas à la manière d'un Michael Moore, dont les documentaires à thèse s'apparentent à des chemins d'uppercuts bien alignés d'où le spectateur ne peut guère s'échapper. Sauper frappe en finesse. Il a l'art de vous restituer son coup d'œil sur ce bas monde sans jugement ni colère. A vous de juger, à vous de vous fâcher.

Pourtant, rien ne semblait prédestiner cet Autrichien à devenir témoin de notre planète. Né au bas de la Streif, la mythique piste de ski de Kitzbühel, il est enfant d'hôteliers.

15) Michel Serfaty a fait dire à son passager qu'il était prêt à le rencontrer à la synagogue. Pour autant, assure-t-il, son chapeau n'est en rien une couverture. «C'est un fait de société. C'est culturel.»

Entre ce juif et les Arabes, c'est une longue histoire. «Je suis né à Marrakech en janvier 1943», raconte-t-il. Famille aisée, très religieuse, père commerçant issu d'une longue lignée de rabbins, mère fille de couturiers du roi, fratrie de douze enfants dont il est le septième. (Libération, 10/12/03)

En outre, ces $\mathrm{SN}$ « nus » passent nettement moins bien que les modifiés comme le suggère le contraste entre (16a-17a) et (16b-17b) ${ }^{11}$ sauf s'ils contiennent un $\mathrm{N}$ dit « de qualité » au sens de Milner (1978) (cf. 18) :

16a) David Peace, 37 ans. L'Etrangleur du Yorkshire, un criminel qui a marqué son enfance, hante l'oeuvre de cet auteur de romans noirs, qui voue un culte aux victimes.

16b) ?David Peace, 37 ans. L'Etrangleur du Yorkshire, un criminel qui a marqué son enfance, hante l'oeuvre de cet auteur.

17a) Quelque 120 personnes, selon la police, ont honoré la mémoire de Brahim Bouarram, dimanche matin, sur le pont du Carrousel à Paris, dix ans jour pour jour après la mort de ce jeune Marocain jeté dans la Seine par des manifestants issus du cortège du Front national.

17b) ?Quelque 120 personnes, selon la police, ont honoré la mémoire de Brahim Bouarram, dimanche matin, sur le pont du Carrousel à Paris, dix ans jour pour jour après la mort de ce Marocain. 
18) Frédéric Beigbeder, le pubard repenti, s'est amouraché de cet «olibrius» et a publié son manuscrit sans barguigner, «entre grands dadais, on se comprend». Ils ont fêté ça au Fouquet's avec une bonne bouteille. attributifs des copulatives sous-jacentes aux SNDP. On sait, en effet, que, pour pouvoir être modifiés, les SN doivent être déterminés par un (vs ø), ce qui correspondrait donc à une copule de type $S N 1$ est un $N 2$ (+mod) et non pasà $S N 1$ est øN2 comme l'illustre les exemples (19) à (20) :

19) Lucie est (ø) escrimeuse

20) *Lucie est (ø) escrimeuse habile

21) Lucie est une escrimeuse (habile)

\subsubsection{DONT LE SN ATTRIBUTIF DÉSIgNE DES QUALIfIANTS INTERNES STABLES}

De la structure de la copulative sous-jacente - SN1 être un N2 - il résulte que, premièrement, les propriétés ainsi prédiquées sont des propriétés stables (vs épisodiques) ${ }^{12}$, comme le rappellent les tests bien connus de Kupferman (1991) présentés sous (22-25), et que, deuxièmement, les référents concernés sont, de ce fait, situés des classes bien établies :

22) Que fait Luc ? Luc est (médecin+*un médecin) (ex. de Kupferman, 1991:66)

23) Ce qui se passe : *Luc est un médecin (ex. de Kupferman, 1991:64)

24) Luc est médecin à Paris depuis 10 ans (Giry-Schneider, $1991: 31$ )

25) *Luc est un médecin à Paris depuis 10 ans (Giry-Schneider, $1991: 31$ )

26) Léa est *une/ø enseignante en ce moment (ex. Kupferman, $1991: 66$ )

Très significativement, les SNDP construits sur la base d'adjectifs comme en (27), dénotent aussi des propriétés stables (cf. les tests de Kupferman, $1991: 63$, rappelés sous (28-32)) :

27) Malgré sa fraîcheur un peu naïve et plutôt bienvenue, il n'ignore pas que si, par miracle, il sort la marque YSL de l'ornière, il lui faudra aussi assumer son exposition dans les médias. Ce grand timide ${ }^{13}$, qui a longtemps rougi avant de se lancer dans une conversation, dit ne pas avoir d'idées sur la façon dont il modifierait alors son comportement.

28) Luc est timide

29) Etre timide est une caractéristique/*un état agréable

30) *Avec Luc de timide

31) *Il est devenu timides toutes sortes de gens

32) *Luc a terminé/débuté timide

Le SN cet élégant de (33) ne constitue une exception qu'en apparence, car il renvoie aussi à une catégorie instituée ${ }^{14}$ :

33) Sa réputation est parfois plus connue que ses refrains. «J'ai la réputation d'avoir une réputation déplorable de prétentieux.» La rumeur s'est installée en effet, agrippée à sa mèche trop soignée, à sa moue renfrognée. «J'ai le défaut d'être très émotif. Ce qui est dangereux, c'est que ça ne se voit pas.» Elle chuchote des coups bas, le raconte tel un Bel Ami, qui fait des chansons pour les dames dont le nom brille ou brilla. Cet élégant n'en serait pas un. La bande des chanteurs trentenaires, Benabar, Delerm... ne peut pas l'encadrer. (Libération, 17/12/03)

Quant aux SN de nationalité tels que (14), (15) et (17) vus plus haut et célibataire (35), octogénaire (36) et trentenaire (37) ${ }^{15}$, ils ne dévient de cette tendance qu'en apparence, dans la mesure où ils sont passibles d'une double interprétation ( $c f$. Kupferman, art. cit. : 67 et seq.), l'une illustrée par (34a) où øN équivaut à un étiquetage « externe » ${ }^{16}$ (tel que fumeur/ non fumeur) visant, dans certaines situations sociales à distinguer, singulariser des 
individus (humains), sans plus ; l'autre, illustrée par (34b), où un $\mathrm{N}$ inscrit le référent «dans une classe de particuliers réunis par un ensemble de propriétés qui font l'objet d'un autre consensus » (Kupferman, $1991: 70)$ :

34a) Paul est Américain (Kupferman, $1991: 70$, ses ex. 71 et 72)

34b) Paul est un Américain

34c) Paul est Américain et Français (double nationalité)

34d) *Paul est un Américain et un Français (contradiction)

34e) Paul est un Américain ou un Français

35) Benicio Del Toro n'a certes aucun signe extérieur du star system. Pas de villa cossue à Berverly Hills, mais toujours le même deux pièces de West Los Angeles où ce célibataire vit comme un étudiant encombré de ses toiles de peintre amateur [...] 36) Vaidelotis le Letton

Architecte de formation, cet octogénaire a connu toutes les étapes tragiques de son pays, stalinisme, occupation nazie, absorption par l'ex-URSS. En septembre, il dira "oui" à l'Union européenne. (Le Monde, 13.06.03)

37) Il a les traits stagnants d'une bouderie adolescente. Le gamin en lui n'est jamais loin. On dirait que l'ennui rôde. Le menace. Le pousse à en découdre avec la chanson d'après. «La chanson, c'est le seul monde où tout est joli. Y a plus de jugements, plus d'ennemis, de craintes, d'échéances du quotidien, y a que des émotions.» Le pousse à en découdre avec la vie d'après.

Ce trentenaire déteste le reflet de sa génération, qui a eu la vie douce au point de se passer d'idéal et de politique, et qui frétille quand voici revenue l'Ile aux enfants. «Moi j'y étais, mais j'y étais pas.» (Libération, 17/12/03)

\subsubsection{Rôle des modifieurs dans les SNDP}

Il reste maintenant à déterminer le rôle des modifieurs, dont la présence quasi obligatoire n'a guère été commentée, à notre connaissance du moins, bien que là encore, les exemples fabriqués exploités par certains auteurs en soient systématiquement dotés :

41) (Jean est venu.) Ce professeur de philosophie...

Ce type toujours optimiste... (Corblin, 1987, 213, son ex. 30)

42) Paul est allé voir l'avocat qui habite dans la vieille ville. Cet avocat de 35 ans ... (Kleiber, 2001, 188, son ex. 13)

3) Mais voilà qu'un beau matin, une vendeuse les sortit de leur boîte pour les essayer à une dame. [...] cette bourgeoise mal attifée elle les mit, fit quelques pas avec ... (Reichler-Béguelin, 1995 : 54, d'après Gripari)

On peut avancer, en première explication, qu'il s'agit notamment d'adjectifs descriptifs ( cf. 38 à 40) et qu'ils ne font que renforcer les caractéristiques internes prédiquées via la phrase copulative sous-jacente. Mais cela ne rend pas compte de leur caractère quasi obligatoire :

38) Avec plus de neuf millions d'entrées, le film reste l'un des plus grands succès de l'histoire du cinéma français et a fixé durablement l'image de cet homme un peu trop gros (quoique sa silhouette ait souvent varié au fil des ans), aux grands yeux naïs, si gentil qu'il parvient à surmonter la méchanceté du reste de l'humanité. Mais ce n'est pas rendre justice à cet acteur étonnant que de se limiter à cette version-là de l'acteur.

39) Ancien élève de l'Ecole Supérieure des Arts Décoratifs de Strasbourg, ce jeune illustrateurtalentueux a de nombreux livres à son actif. Il viendra dédicacer ses derniers ouvrages. Ses dessins originaux seront exposés à la librairie jusqu'au 4 avril 2005.

40) «Je suis encore en train de découvrir mon poste : ma satisfaction vient surtout de mon travail», se justifie cet employé modèle qui a toujours "aimé» ses patrons. Aujourd'hui, il se pose des questions sur son nouveau statut: «Y a beaucoup de patrons qui sont cons, non?» 

puisque le SN présuppose à la fois l'existence des Allemands, ce qui permet en soi l'emploi du SN. Mais comme, avec les SN démonstratifs, la présupposition d'existence est, selon Kleiber (1984: 67), une conséquence de leur emploi, les présupposés tournent, en quelque sorte, « en rond » :

Il existe des Allemands $\rightarrow$ Ce $\mathrm{x}$ est un Allemand $\rightarrow \mathrm{Il}$ existe des Allemands

Il suffit, en revanche, de modifier le SND, comme dans (43) ${ }^{19}$ pour constituer, dans l'ensemble des Allemands dont est présupposée l'existence, un sous-ensemble dont il est possible, comme le suggère Picabia, d'asserter ou de nier l'existence: «les allemands maladroits » ou les «allemands borgnes » partitionnant l'ensemble des allemands en « allemands non maladroits » ou « allemands non borgnes »:

43) Un chasseur est arrivé hier; cet Allemand (maladroit+borgne) a manqué tous ses tirs

Cela serait du reste congruent avec le sens du démonstratif dont Kleiber (1984: 76) souligne la dimension partitive :

Ce $\mathrm{N}$ présuppose l'existence d'autres $\mathrm{N}$, c'est-à-dire d'autres tels et tels (ou d'une autre partie de N)

Le cas des «noms de qualité » qui peuvent figurer «nus » dans les SNDP ${ }^{20}$ fournira un argument supplémentaire. Comme l'a démontré Milner (1978: 174 et seq.), ces N ne renvoient pas à des classes. Outre les arguments avancés par l'auteur lui-même (pp. 201-202), on peut invoquer la difficulté à dire (44) et (45) qui prouve l'impossibilité d'identifier des $\mathrm{X}$ correspondant à une catégorie d'imbéciles ou de situer des occurrences par rapport à leur adéquation à la classe via des enclosures (une sorte, une espèce de). Il est d'ailleurs remarquable que des adjectifs comme $\mathrm{vrai}^{21}$ qui ont également cette fonction, la perdent dans l'environnement des $\mathrm{N}$ de qualité pour signifier l'intensité : 
44) Ceci est un imbécile

45) Aujourd'hui j'ai vu trois imbéciles

46) Paul est un (vrai+parfait+sacré) imbécile

47) Paul est une (sorte+espèce) d'imbécile

48) c'est une bande ${ }^{22}$ d'imbéciles vs c'est un *(ensemble+un groupe) d'imbéciles

$$
\text { 202): }
$$

Comme pour les pronoms personnels, il n'y a pas de classe des «imbéciles », hors

les circonstances où un sujet articule expressis verbis le mot lui-même.

leur emploi en anaphore démonstrative prédicative ne s'assortit pas, comme plus haut, d'un double présupposé. L'article démonstratif remplit alors normalement, si l'on peut dire, sa fonction en ne présupposant simplement que le x en cause est un imbécile.

\subsection{Premier bilan}

$\mathrm{Au}$ terme de cette première partie, nous avons fait valoir un certain nombre de contraintes prohibant l'emploi «tous azimuts» des SNDP. Nous ralliant à la nécessité d'une relation de type être entre les $\mathrm{N}$-source et anaphorique, nous avons fait état d'arguments plus inédits dans le but de préciser cette relation. Premièrement, suivant les suggestions de Van Peteghem, nous avons étayé l'idée que la relation serait une copule de type prédicationnel/classificatoire, dont le SN2 serait déterminé par un et systématiquement modifié. Deuxièmement, nous avons motivé ces modifications en arguant, d'une part, d'un «choc » dans les couches présuppositionnelles du SN nu que seuls seraient à même d'atténuer les modifieurs, en parfaite congruence avec le sens partitif de l'article démonstratif. Ceci étant, maintenant que sont posées des contraintes ${ }^{23}$ au niveau "interne » du SNDP, il s'agit d'envisager leurs répercussions au niveau du discours.

\section{Contraintes « externes » liées à l'environnement discursif des SN démonstratifs prédicatifs}

Pour être permissifs, les auteurs défendant un emploi relativement libre des SNDP n'en reconnaissent pas moins l'incidence probable qu'elles exercent au niveau du discours ou réciproquement. Témoin cette citation :

Il est assez probable qu'en fonction des références virtuelles impliquées, des mécanismes sémantiques ultérieurs complètent ces reclassifications et les évaluent selon le degré de motivation qu'il est possible de leur associer dans un contexte. (Corblin, 1987, 212, nous soulignons)

De fait, il suffit de compléter certains des exemples proposés dans la littérature (p.e. 48), pour se rendre compte que l'acceptabilité des SNDP est très liée au cotexte, comme le suggère le contraste entre (49) et (50), d'une part et, d'autre part (51) et (52) :

48) (Jean est venu.) Ce professeur de philosophie...

Ce type toujours optimiste... (Corblin, 1987, 213, son ex. 30)

49) ?(Jean est venu.) Ce professeur de philosophie, pour une fois, m'a offert des fleurs

50) (Jean est venu.) Ce professeur de philosophie, pour une fois, ne nous a pas bassinés toute la soirée avec la métaphysique de Kant

51) ?(Jean est venu.) Ce type toujours optimiste travaille dans l'informatique 
52) (Jean est venu.) Ce type toujours optimiste se révèle, en toute occasion, un convive très agréable

En quoi le discours et les SNDP se contraignent-ils réciproquement? C'est ce que nous allons essayer de démêler...

\subsection{LE MODE DE DONATION DÉfINI VS INDÉfINI DE LA SOURCE DU SNDP}

Une série de contraintes à caractère configurationnel a été mise au jour par Kleiber (1988, 2001) et Kleiber, Patry \& Ménard (1990, 134-135). Elles proviendraient du mode de donation du référent-source qui exerce un impact sur la recevabilité des SNDP subséquents. Selon le constat établi par les auteurs, les $\mathrm{SN}$-sources à caractère "défini » (de type le $\mathrm{N}$ ou $\mathrm{N}$ propre) autoriseraient des enchaînements prohibés par les $\mathrm{SN}$ indéfinis :

Le problème mérite d'être étendu aux antécédents hyperonymiques définis. Nos informateurs ont trouvé < (53) [dans notre numérotation] > moins anormal que les énoncés à hyperonymes indéfinis mais ont encore noté que la prédication hyponymique de livre par roman leur paraissait injustifiée :

53) Il y a sur la table le dernier livre de Françoise Sagan. Ce roman raconte...

Avec un nom propre comme antécédent, les choses sont plus faciles comme en témoignent des exemples classiques tels que $<(54)$ [dans notre numérotation] $>$ :

54) À 60 ans, Paul Dubois se retira dans son village. Cet ancien coureur cycliste ...

Cette contrainte est également révélée par lecontraste entre (55) et (56):

55) *Paul est allé voir un avocat. Cet avocat de 35 ans ... (Kleiber, 2001, 187, son ex.

11)

56) Paul est allé voir l'avocat qui habite dans la vieille ville. Cet avocat de 35 ans ...

(Kleiber, 2001, 188, son ex. 13)

51 Les raisons invoquées par l'auteur sont les suivantes:

La différence réside dans la présentation du référent de l'expression antécédent. Quand il est présenté au moyen d'une expression définie (ou terme singulier tel que nom propre, description définie, description démonstrative, etc.), il apparaît comme un référent connu autrement que par sa seule appartenance à la classe des $\mathrm{N}$, de telle sorte que l'information (nouvelle ou non pour l'interlocuteur) peut être acceptée comme étant en somme une information disponible ou inférable à partir de la présentation définie (ou particulière) faite du référent. $\mathrm{Si}$, au contraire, il s'agit d'un antécédent indéfini, comme dans le cas de un avocat (...) la seule connaissance que nous en ayons est le fait de son appartenance à la classe des N. Les seules inférences auxquelles donne lieu une telle présentation sont alors celles qui sont liées à son appartenance à la classe des avocats (...), c'est-à-dire (...) celles qui découlent d'une connaissance prototypique ou stéréotypique ou encore connaissance par défaut des membres de la classe N concernée. (G. Kleiber, 2001, 187-188, les gras sont de nous)

Ce point est capital car il invite à dissocier les énoncés à source définie de ceux où elle ne l'est pas et permet de comprendre la gradation établie dans les énoncés ci-dessus. La reprise par ce roman est envisageable dans (53) parce que l'on sait que F. Sagan est romancière et que sa production écrite se constitue pour l'essentiel de romans. De même, le fait de savoir que P. Dubois a été coureur cycliste ( $c f .(54))$ est lié aux connaissances extra-linguistiques que l'on a (ou non) du référent ainsi dénommé. Est-ce à dire que, dans le cas des sources définies, il n'y a plus rien qui bride les SNDP ? Rien n'est moins sûr car il reste à expliquer pourquoi l'enchaînement (57) ainsi complété, passe mal : 
57) ? À 60 ans, Paul Dubois se retira dans son village. Cet ancien coureur cycliste adore les andouillettes ${ }^{24}$ exploitées par les partisans des SNDP «tout venants » dans la mesure où dans beaucoup de leurs exemples la source est définie, ce qu'illustrent quelques exemples repris de Corblin (op. cit.) :

58) Les ouvriers considérèrent le plancher du wagon et décidèrent que pour une nuit, on pouvait se satisfaire de ce lit (F. Corblin, 212, son ex. 27)

59) (La jeune fille était charmante) mais il fut impossible de faire sourire ce glaçon. (Corblin, 213, son ex. 32)

60) (Pierre tint bon) et ce roc ne bougea pas d'un pouce (Corblin, 213, son ex. 33)

Ces contraintes «discursives » étant posées, il reste à déterminer s'il n'y a pas moyen d'introduire, malgré un SN indéfini, des contenus moins « codifiés » et si, en cas de source définie, il n'y a pas matière à préciser le rôle que joue le cotexte.

\subsection{Ouvertures et fermetures}

Les éléments de réponse que nous fournirons à cette question seront distingués selon le mode de donation, défini ou non, du SN-source.

\subsubsection{LA SOURCE EST INTRODUITE VIA UN N}

Dans l'hypothèse où, en présence d'une source indéfinie, les contenus du SNDP sont limités aux informations stéréotypées liées au $N \mathrm{du} \mathrm{SN}$, les possibilités de prédication «inédites» via le SN démonstratif peuvent être envisagées sous deux conditions au moins.

\subsubsection{La mention dans le cotexte d'une " source de savoir"}

Soit l'exemple (61) dont le SN-source est indéfini :

61) ? Un homme entra dans un bar. (Ce molosse barbu+ cet italien bilingue+cet intellectuel de gauche+ce fan de David Bowie+ce notaire+ Ce Breton) se dirigea d'un pas décidé vers le comptoir

Rien dans les environnements gauche et droit du SNDP ne motive les apports informatifs entre parenthèses. Il suffit, en revanche, d'introduire dans le cotexte une «source de savoir ", réelle ou potentielle, pour que certaines informations passent beaucoup mieux :

62) Un homme entra dans un bar. Le patron dévisagea avec curiosité (ce molosse barbu+ ${ }^{*}$ cet italien bilingue).

63) Je venais de m'installer à un table lorsqu'un homme entra dans le bar. (Ce barbu/molosse/ivrogne/vieillard) se dirigea d'un pas hésitant vers le comptoir

De fait, l'introduction d'une sorte de «sujet de conscience » dans le cotexte, le patron du bar dans (62), ou un sujet-énonciateur à la première personne, dans (63), rend possible l'introduction d'informations non conventionnelles sur le référent ${ }^{25}$. Maintenant les énoncés ci-dessus montrent que ce principe ne permet pas tout.

\subsubsection{Nature et accessibilité des informations}

Encore faut-il prendre en compte la nature des informations qui sont accessibles à ce sujet de conscience dans la situation dénotée par le discours. Dans (62) et (63), les SNDP 
qui passent sont ceux dont les informations sont immédiatement accessibles, notamment par la vue - en l'occurrence la taille, la tranche d'âge - autrement dit, elles sont, par défaut, disponibles dans la plupart des situations mettant en présence des individus. Dans (64), le fait que le personnage passe sa commande rend plausible le SNDP cet italien bilingue, puisqu'en parlant il rend disponibles des informations sur un éventuel accent, donc la nationalité, etc. :

64) Un homme entra dans un bar et commanda une Budweiser au comptoir. Le patron dévisagea avec curiosité (ce molosse barbu+ cet italien bilingue).

61 En revanche, les reprises de (65) et (66) restent peu plausibles car les informations délivrées par les SNDP ne sont pas, sinon peu, accessibles étant donné les circonstances dénotées par le cotexte. Seule, l'intervention d'un "narrateur omniscient", susceptible d'anticiper la suite des événements, justifie a fortiori la prédication d'informations sans cela inaccessibles ${ }^{26}$ :

65) ?Un homme entra dans un bar et commanda une Budweiser au comptoir. Le patron dévisagea (cet intellectuel de gauche+ce fan de David Bowie+ce représentant de commerce+ Ce Charentais) se dirigea d'un pas décidé vers le comptoir.

66) Un homme entra dans un bar et commanda une Budweiser au comptoir. Cet intellectuel de gauche allait bientôt convaincre le patron de voter contre la constitution européenne.

\subsubsection{LA SOURCE EST INTRODUITE VIA LE N/NPR}

En cas de source définie, la connaissance du référent étant acquise - ou susceptible de l'être -, suivant la sugestion de Kleiber, par d'autres voies que le cotexte, les formes de SNDP semblent effectivement se soustraire à toute forme de contrainte. C'est du reste ce qui explique que, dans les portraits journalistiques exploités en partie pour cette étude, les rédacteurs ne se privent pas de diffuser " par la bande » toutes sortes d'informations y compris les plus « exotiques » sans qu'on y trouve rien (ou quasi) à y redire:

67) Voici Piège, revêtu de sa propre casaque, lancé dans la course aux étoiles. Car les palaces viennent de réaliser que la cotation Michelin est cruciale en ces temps de clientèle nomade. Le Georges-V et le Plaza affichent trois macarons. Le Bristol, deux. Le Crillon n'en a qu'un et se doit de monter en gamme. Pour autant, Piège ne se soumet pas aux vieux diktats du luxe. Ce salarié de haut niveau, qui refuse d'en donner le montant (10 000 euros mensuels ?), n'abjure ni son époque, ni ses origines. Il se flatte d'appartenir à «la première génération qui a connu les fraises tagada et les carambars». D'où les serveurs en smoking-blouse, l'autorisation du sans-cravate, les menus-déjeuner à 70 euros. Ou, également, son renvoi dos à dos «de la droite qui fait du populisme et de la gauche qui devient libérale». Pourtant, ce petit-fils d'un agent SNCF adhérent CGT et d'un maquisard du Vercors qui ressemblait à Gabin, jongle avec les 35 heures sans les maudire comme tant d'autres. Et il a fait campagne auprès de ses commis pour qu'ils aillent voter contre Le Pen, en 2002. Pas baptisé, il n'est pas franc (Libération, 14/01/05)

68) Marc Jacobs, 41 ans, Américain, styliste de sa marque et directeur artistique chez Vuitton, qu'il a contribué à relancer. Doué en affaires, il se pose plutôt en rebelle du luxe.

Double étiquette

Il y a des préoccupations plus angoissantes, mais lui non plus ne sait jamais quoi se mettre quand il sort, redoute le geste absurde de se déshabiller pour se rhabiller, ne va pas seul à des fêtes, et surtout pas à des soirées où il ne connaît personne. Mais c'est improbable. Le soir du nouvel an, il en a profité «pour travailler» et s'est nourri d'un «sandwich au poulet», mangé sur le canapé, chez lui, devant la télé. Plus tard, à l'aube, il est sorti, doit-on à la vérité. Bref, cet homme qui porte un pull 
peluché bleu marine Comme des garçons et des sneakers blanches est exactement comme vous. (Libération, 04 mars 2005)

Pour autant, cela ne va pas sans provoquer quelques couacs - c'est le cas de le dire avec dans (8), vu en introduction, le SN ce virtuose de l'accordéon :

8) Daniel Libeskind

Bâtisseur d'espoir

Fils d'immigrés juifs polonais, cet architecte a remporté le concours pour la reconstruction du World Trade Center.

Né en Pologne après la Seconde Guerre Mondiale, Daniel Libeskind y a vécu l'antisémitisme du régime communiste après que ses parents avaient subi celui des nazis. Sa famille a rapidement émigré en Israël, où elle s'est vite lassée de la vie dans les kibboutzim avant de partir pour les États Unis. De quoi donner le goût du nomadisme - avec quatorze déménagements en trente-cinq ans ! - à ce virtuose de l'accordéon! (TV Hebdo, 2005)

Apparemment ce n'est pas en soi le contenu informatif qui est en cause, tout aussi " pointu » à cet égard que celui des SNDP de (67) et (68).

L'hypothèse que l'on peut formuler pour rendre compte de cette incongruité tient précisément à la nature prédicative du SN démonstratif. En effet, dans la mesure où il y a prédication, on peut penser que celle-ci se soumet aux contraintes auxquelles sont assujetties les prédications en général, à savoir le respect d'un principe de cohérence. En temps «normal», celui-ci concerne deux propositions ou segments adjacents (ou hiérarchisés). Mais, en présence d'une anaphore prédicative, ce principe semble naturellement s'instaurer entre le SNDP et la prédication de sa proposition-hôte. Le lien avec le cotexte gauche serait moins important compte tenu du sens du SN démonstratif « qui fait intervenir le contexte d'énonciation de sa propre occurrence » (cf. Kleiber, 1988, 68) abstraction faite des circonstances d'évaluation qui en motiveraient l'emploi. Ainsi, l'énoncé (8) se paraphraserait-il comme suit :

69) ? $\left(p_{1}\right)$ La famille de D. Libeskind a beaucoup « voyagé » $\left[_{(p 2)}\right.$ ce $x$ identifié comme

étant $\mathrm{D}$. Libeskind est un virtuose de l'accordéon $]^{27}\left(\mathrm{p}_{3}\right)$ (Il) a le goût du nomadisme.

Or, comme cela a été démontré dans les travaux sur la cohérence textuelle, la succession de deux propositions invite l'interprète à établir entre celles-ci un lien, le plus « naturel » - et le plus fort - étant celui de causalité, le plus faible étant un lien d'analogie. Dans le cas des SNDP, la prédication est, si l'on peut dire, « encapsulée » dans la proposition. En d'autres termes, les deux prédications seraient plus intimement liées qu'elles ne le sont en (69). De sorte que l'on peut se demander si ceci n'oblige pas à établir le lien de cohérence le plus fort entre les deux, un lien de causalité donc.

Par ailleurs, d'un point de vue informationnel, le SNDP est ambivalent. En effet, l'information nouvelle se trouve présupposée via le SN, autrement ce qui est d'un point de vue informatif totalement nouveau est dit présenté comme connu. Ceci pourrait orienter le rapport de causalité, ainsi que le suggère Forsgren (2000) à propos d'une structure proche :

70) Jean, cet imbécile, a cassé la tasse (M.Forsgren, 2000 : 38)

ii) SNDP prédicat

\section{Cause conséquence}

On notera ainsi, dit l'auteur, la discordance énonciative entre la propriété imbécile, fournie sous forme d'un catégorématique présupposant, et le prédicat premier a cassé la tasse, fourni sous forme d'assertion, de posé. On en arrive ainsi à se demander si le locuteur choisit la formulation en question parce qu'il considère que Jean est un imbécile 
parce qu'il vient de casser la tasse, ou si, ce qui constitue mon hypothèse, c'est l'inverse : Jean vient de casser la tasse parce que c'est un imbécile. Ainsi le fait de casser la tasse est présenté comme une conséquence de l'imbécillité de Jean. Par le SN présupposant cet imbécile, on suggère fortement - stratégie rhétorique ! - que Jean a commis d'autres actes imbéciles auparavant. (Forsgren, $2000: 38$ )

Dans cette optique, on interpréterait à partir de (67) que c'est parce que Piège est petit fils d'un adhérent CGT et d'un maquisard, donc plutôt « humaniste de gauche », qu'il jongle avec les 35 heures ou, dans (68) que c'est parce qu'il porte les habits de «monsieur-toutle-monde » (et non pas ceux de la marque pour laquelle il travaille entre autres) que M. Jacobs est comme le lecteur de l'article :

67a) Pourtant, ce petit-fils d'un agent SNCF adhérent CGT et d'un maquisard du Vercors qui ressemblait à Gabin, jongle avec les 35 heures sans les maudire comme tant d'autres.

68a) Bref, cet homme qui porte un pull peluché bleu marine Comme des garçons et des sneakers blanches est exactement comme vous.

Or, ce genre de rapport est nettement plus difficile à établir pour (8) (du moins pour nous) entre le goût du nomadisme et celui de l'accordéon.

\section{Conclusion}

73 Au terme de cette étude, nous avons fait valoir deux ordres de contraintes qui pèsent sur l'emploi des SNDP. La première concerne le niveau du SN lui-même. Nous avons essayé d'expliciter la relation copulative qui lie le $\mathrm{SN}$ anaphorique à sa source en montrant qu'il s'agit d'une relation copulative prédicationnelle du type SN1 est un N2+modifieur où le $\mathrm{N}$ renvoie à une classe de propriétés «internes » et où le modifieur motive l'opération de partition impliquée par le sens de l'article démonstratif.

74 La seconde série de contraintes oblige à prendre en considération le mode de donation, défini ou indéfini, de la source de l'anaphorique démonstratif, qui, le cas échéant, limite le type d'informations susceptibles de figurer dans le SNDP. Deux principes en fait régulent l'information :

- un principe de pertinence qui rend compte des modalités selon lesquelles des informations non stéréotypées peuvent être le cas échéant, accessibles ;

- un principe de cohérence qui repose sur le rapport de causalité liant le SNDP et son prédicat : c'est parce que $x$ est un SN2 que $p$ où $p$ n'est qu'une des manifestations du caractère véhiculé par SN2, selon l'hypothèse de Forsgren.

Incidemment sont apparues des «passerelles » qui rattachent les SNDP, d'une part, à la question de la prédication seconde et d'autre part, aux autres formes de relations "attributives" qu'instaurent les appositions, constructions détachées et autres qui montrent, une fois encore, que les perspectives parfois insoupçonnées qu'ouvre la question des anaphores. 


\section{BIBLIOGRAPHIE}

CORBLIN F. (1987) Indéfini, défini et démonstratif, Genève-Paris, Droz.

DE MULDER W. (1997) Les démonstratifs : des indices de changement de contexte, in N. Flaux, D. Van de Velde et W. De Mulder (éds), Entre général et particulier : les déterminants, 137-200.

DE MULDER W. (2001) Peut-on définir les SN démonstratifs par leurs contextes, in H. Kronning et al., Langage et référence. Mélanges offerts à K. Jonasson à l'occasion de ses soixante ans, Uppsala, Acta Universitatis Upsaliensis, 115-123.

FORSGREN M. (1988) Apposition adnominale : déterminants et ordre des constituants, Travaux de linguistique 17, 137-155.

FORSGREN M. (2000), Apposition, attribut, épithète : même combat prédicatif ?, Langue française $125: 30-45$.

GIRY-SCHNEIDER J. (1991) L'article zéro dans le lexique-grammaire des noms prédicatifs, Langages $102: 23-35$.

KLEIBER G. (1984) Sur la sémantique des descriptions démonstratives, Lingvisticae Investigationes VIII, 1, 63-85.

KLEIBER G. (1986) Déictiques, embrayeurs, «token reflexives », symboles indexicaux, etc. : comment les définir? L'information grammaticale, 30, 3-21.

KLEIBER G. (1986) Pour une explication du paradoxe de la reprise immédiate, Langue française 72, 54-79.

KLEIBER G. (1988) Sur l'anaphore démonstrative, in G. Maurand (éd.), Nouvelles recherches en grammaire, Albi, Université Toulouse le Mirail, 51-74.

KLEIBER G. (1989) Les démonstratifs de près et de loin, Strasbourg 2, Anaphore \& Deixis 2.

KLEIBER G. (1994) Anaphores et pronoms, Louvain-la-Neuve, Duculot.

KLEIBER G. (2001), L'anaphore associative, Paris, Seuil.

KLEIBER G. (2003) Adjectifs démonstratifs et point de vue, Cahiers de Praxématique 41, 33-54.

KLEIBER G., PATRY R., MÉNARD N. (1990) « L'anaphore associative : dans quel sens roule-t-elle?, in C. Schnedecker, M. Charolles, G. Kleiber, J. David (éds), L'anaphore associative, Paris, Klincksieck. KUPFERMAN L. (1991) Structure événementielle de l'alternance Un/ø devant les noms humaines attributs, Langages $102: 52-75$.

LEGALLOIS D. (2002) Incidence énonciative des adjectifs vrai et véritable en antéposition nominale, Langue française 136 : 46-59.

LEGALLOIS D. (2004) Cohésion lexicale et réseaux phrastiques dans la constitution de textes expositifs, in S. Porhiel et D. Klinger (éds), L'unité texte, Association Perspectives, 171-201.

MARANDIN J.-M. (1986) Ce est un autre. L'interprétation anaphorique du SN démonstratif, Langages 81, 75-89.

MILNER J.-C. (1978) De la syntaxe à l'interprétation, Paris, Seuil. 
MILNER J.-C. (1982) Ordres et raisons de langue, Paris, Seuil.

PICABIA L. (1991) Articles zéro et structures apposées, Langages 102 : 88-102.

PICABIA L. (2000) Appositions nominales et déterminant zéro, Langue française 125 : 71-89.

REICHLER-BÉGUELIN M.-J. (1995) Alternatives et décisions lexicales dans l'emploi des expressions démonstratives, Pratiques 85, 53-87.

SCHNEDECKER C. (à par. a) Les chaînes de référence dans les portraits journalistiques, Travaux de linguistique 51.

SCHNEDECKER C. (à par.b) SN démonstratifs prédicatifs : qu'est-ce qui limite leur apport informatif ?, Langue française.

SERIOT P. (1988) L'anaphore et le fil du discours (sur l'interprétation des nominalisations en français et en russe) in H. Nølke (éd.), Opérateurs syntaxiques et cohésion discursive, Nyt Nordisk Forlag Arnold Busck, 147-160.

VAN PETEGHEM M. (1991) Les phrases copulatives dans les langues romanes, Wilhelmsfeld, Egert.

VAN PETEGHEM M. (1998) Sur le contenu lexical des descriptions définies et démonstratives, in M. Forsgren et al. (éds), Prédication, assertion, information, Uppsala, Acta Universitatis Upsaliensis, 569-578.

\section{NOTES}

1. Pour qui, rappelons-le, les unités démonstratives ne sont pas anaphoriques. (cf. op. cit., 25)

2. On rencontre en effet ce genre de problème avec les $\mathrm{SN}$ définis : Lors d'une soirée au cours de laquelle alcool et cannabis sont consommés, Julien se rend à un rendez-vous fixé par un dealer. Lorsque ce dernier est retrouvé mort, l'adolescent est accusé de meurtre et finit par avouer. (TV Hebdo, 13/10/2004)

3. Répertoirés dans Schnedecker (à par. b).

4. Voir aussi, p. 59.

5. Voir les discussions menées par Kleiber (1988) et De Mulder (1997) à ce propos.

6. Qui exclut la relation d'identité du type: "J'ai rencontré madame Onassis. Cette Jackie Kennedy prenait l'avion pour Londres. "

7. Qui met en relation deux SN de même force référentielle (cf. art. cit., 572)

8. Ce type de relation conviendrait aux $\mathrm{SN}$ démonstratifs et définis selon l'auteur (art. cit.: 572-573).

9. Qui nous semble, d'après les critères indiqués dans l'ouvrage dédié aux constructions copulatives du même auteur (1991) plutôt identificationnelle. Van Peteghem (1991 : 25) reconnaît d'ailleurs qu'il peut y avoir ambiguité entre les deux types de copules notamment quand le SN2 attribut est défini ou indéfini comme cela est vraisemblablement le cas ici.

10. À la différence des $\mathrm{SN}$ définis qui le sont très fréquemment ( $c f$. Schnedecker, à par.a).

11. Sauf à invoquer un emploi contrastif du SNDP, qui est illustré par exemple dans l'énoncé (15) où ce juif s'oppose aux Arabes.

12. Ces propriétés n'ont pas de dimension processuelle (23) et sont non pas agentives (22) mais statives et qu'elles sont abstraites de tout ancrage spatio-temporel ponctuel (cf. 24 et 25) ce qui a pour corollaire qu'ellesne se laissent pas « borner »).

13. On a là manifestement une dénomination dans la mesure où timide n'est pas gradable: ?Un très grand timide, et où grand timide est difficile en position d'attribut : Il est grand timide. 
14. Le Petit Robert atteste son emploi, quoique vieilli, en tant que nom. Merci à D. Legallois d'avoir attiré notre attention sur ce point.

15. Les trois derniers sont susceptibles de renvoyer autant à l'étiquetage socio-culturel dont parle Kupferman ( $c f$. les formulaires d'état-civil, de recensement, etc. où il faut préciser l'âge et l'état civil) qu'à une classe dotée de ses prototypes (cf. les SN figés de vieux célibataire, célibataire endurci où les adjectifs insistent sur l'appartenance à la catégorie via la longévité, etc.).

16. De là viendrait une référence « à la classe comme à un objet brut, encore indissocié » où le SN se trouverait « déposé sur elle comme une étiquette » (ibid.).

17. Pour qui, rappelons-le, les unités démonstratives ne sont pas anaphoriques. (cf. op. cit., 25)

18. Et ce, sur la base d'un parallélisme dans le fonctionnement des phrases existentielles impersonnelles et des apposées: \#Il existe des singes $\approx$ \#Chita, singe ... que nous étendrions volontiers aux SNDP.

19. Qui pour être meilleur que (1) n'est pas pour autant totalement recevable, $c f$. infra.

20. Le fait est aussi qu'ils ne supportent pas sans contraintes (cf. Milner, 1978: 207 et seq.) les modifieurs qualifiants du type de gentil, coquet, etc.

21. Cf. Legallois, 2002.

22. Regroupement occasionnel de personnes ayant des points communs. (Petit Robert).

23. Nous ne prétendons pas épuiser la matière...

24. Si, comme nous le suggère D. Legallois, l'emploi de l'imparfait améliore quelque peu l'énoncé : À 60 ans, Paul Dubois se retira dans son village. Cet ancien coureur cyclisteadorait les andouillettes, il ne le rend pas complètement naturel pour autant.

25. Reichler-Béguelin tire argument de la reprise hyponymique de l'exemple suivant: "C'est ainsi qu'au Rivier (domaine de la présidente) de magnifiques oiseaux blancs ont pu être observés pendant 15 jours. Ils étaient très grands, très nombreux, dotés d'un long et gros bec jaune-rouge qu'ils glissaient dans l'eau pour pêcher, en avançant à pied dans la vasière. Ces « spatules » ont pu être vues par tous ceux qui ont emprunté les promenades piétonnières organisées pour le public » (nous soulignons) pour réfuter la contrainte posée par Kleiber et al. (1990) sur le SN indéfini en disant (note 37, p. 73) : « Il est bien possible qu'une étude empirique permette de confirmer cette intuition, mais l'exemple qu'on vient d'analyser montre bien que le rappel spécifiant concerne aussi des objets-de-discours introduits sous forme indéfinie ». Or, si la source est effectivement un SN indéfini, son référent est appréhendé par des sujets de conscience (cf. les verbes en italiques dans l'exemple), ce que l'auteur souligne sans établir la corrélation.

26. C'est ce que suggère, du reste, Reichler-Béguelin : «les anaphores de cette catégorie sont parfois le support de subtils faits de polyphonie, quand la reclassification de l'objet, n'est plausible qu'à condition d'être assignée au point de vue d'un personnage, en fonction de ce que l'on sait de lui. [...] Il ne fait pas de doute que la recevabilité des anaphores démonstratives est en partie fonction de ces phénomènes. $(1995,75)$.

27. Les crochets et le $\mathrm{P}_{2}$ en indice indiquent qu'il s'agit d'une forme de prédication seconde.

\section{RÉSUMÉS}

Dans cette étude, nous faisons valoir deux ordres de contraintes qui pèsent sur l'emploi des syntagmes nominaux démonstratifs prédicatifs (SNDP) i.e. véhiculant de l'information nouvelle, par rapport à celle que donne leur antécédent et/ou son contexte d'occurrence. La première 
concerne la relation copulative liant le $\mathrm{SN}$ anaphorique à sa source, qui est prédicationnelle et dont le $\mathrm{SN}$ attribut (de forme N2+modifieur) renvoie à une classe de propriétés « internes » et où le modifieur motive l'opération de partition impliquée par le sens de l'article démonstratif. La seconde série de contraintes prend en compte le mode de donation, défini ou indéfini, de la source de l'anaphorique démonstratif, qui, le cas échéant, limite le type d'informations susceptibles de figurer dans le SNDP. Deux principes en fait régulent l'information: 1) un principe de pertinence qui rend compte des modalités selon lesquelles des informations non stéréotypées peuvent être le cas échéant, accessibles ; 2) un principe de cohérence qui repose sur le rapport de causalité liant le SNDP et son prédicat.

In this study, we put forward two constraints which limit the use of the predicative demonstrative NP (PDNP) i.e. conveying new information, compared to that given by its antecedent and/or the context. The first concerns the relation between the anaphoric SN and its source, which is "predicational» (in the sense of Van Peteghem, 1991) and whose SN attribute (of N2+modifier form) returns to a class of inherent properties and where the modifier justifies the partition implied by the demonstrative. The second series of constraints takes into account the role of the definite vs indefinite determinant of the antecedent of the PDNP, which limits the informational content. Actually, two principles control it: 1) a principle of relevance which explains why not «stereotyped » information can be accessible ; 2) a principle of coherence, in particular relation of causality between the PDNP and its predicate.

\section{AUTEUR}

\section{CATHERINE SCHNEDECKER}

Université Marc Bloch, Strasbourg 2, LILPA EA 1339, Scolia 